\title{
Performance analysis of 5-ary MUSA and SCMA for uplink transmission
}

\author{
Esraa Mosleh Eid ${ }^{1}$, Mostafa M. Fouda ${ }^{2}$, Adly S. Tag Eldien ${ }^{3}$, Mohsen M. Tantawy ${ }^{4}$ \\ ${ }^{1,2,3}$ Electrical Engineering Department, Faculty of Engineering at Shoubra, Benha University, Egypt \\ ${ }^{4}$ Network Planning Department, National Telecommunication Institute, Egypt
}

\begin{tabular}{l}
\hline Article Info \\
\hline Article history: \\
Received Jul 9, 2018 \\
Revised Nov 18, 2018 \\
Accepted Feb 4, 2019 \\
\hline
\end{tabular}

\section{Keywords:}

5-ary codes

Grant-free access

Multi user shared access

(MUSA)

Sparse code multiple access

(SCMA)

User overloading

\section{Corresponding Author:}

Esraa Mosleh Eid,

Electrical Engineering Department,

Faculty of Engineering at Shoubra,

Benha University, Cairo, Egypt.

Email: esraa.soliman@feng.bu.edu.eg

\begin{abstract}
Multiple access is one of the core technologies of wireless communications, which enables wireless base stations to deal with a large number of different users and provide the service for each of them at the same time. Meeting the $5 \mathrm{G}$ challenges, non-orthogonal multiple access is one of the main concerned points in $5 \mathrm{G}$ technologies. In this paper, two types of non-orthogonal multiple access schemes, namely, 5-ary Multi User Shared Access (5-ary MUSA), which is a our proposed scheme of MUSA that uses a new type of spreading codes called 5-ary codes, and Sparse Code Multiple Access (SCMA), are studied. The Bit Error Rate performance (BER) analysis is shown for different user overloading for uplink systems. Simulation results provide a clear view on the effect of increasing the number of users for the same system parameters.
\end{abstract}

Copyright $(2019$ Institute of Advanced Engineering and Science. All rights reserved.

\section{INTRODUCTION}

In the upcoming $5 \mathrm{G}$ network, it is expected that it can hold enormous number of applications and terminals. One of the most important $5 \mathrm{G}$ requirements is that it should support enormous connectivity [1] with a huge number of devices. The new standard is expected to be implemented in 2020; consequently at this phase a lot of research is being carried out. $5 \mathrm{G}$ systems should be able to support a rate of several 10's of megabits per second serving 10000's of users and a latency of $1 \mathrm{msec}$ [2]. Non-Orthogonal Multiple Access (NOMA) schemes are recommended as the proper solution to raise the number of connected terminals for restricted number of resources [3]. Unlike traditional orthogonal multiple access techniques, NOMA introduces some controllable interference to implement overloading with increasing the receiver complexity leading to more spectral efficiency and enormous connectivity [4].

Different non-orthogonal multiple access schemes are suggested for $5 \mathrm{G}$ communication networks, such as Multi User Shared Access (MUSA) and Sparse Code Multiple Access (SCMA) [5] and others. MUSA is a one of the $5 \mathrm{G}$ multiple access scheme that is suggested by ZTE. MUSA employs the characteristics of suitable spreading sequences and Successive Interference Cancellation (SIC) receiver [6]. At the transmitter side, user's data are spreaded first by using one of the special spreading sequences, and then they are overlapped and transmitted through the channel. At the receiver side, an SIC receiver is used to recover the data of each user.

In $[7,8]$, the authors introduced SCMA as one of the new multiple access scheme. In SCMA, incoming data flow from different users are straight mapped to code words of diverse multi-dimensional codebooks; with 
each codeword appears as a spread transmission layer. The sparsity of code words makes the detection appropriate through refined message passing algorithm (MPA). In [4], the authors compared the performance of three NOMA schemes, SCMA, MUSA (3-ary type), and Pattern Division Multiple Access (PDMA). The results show that SCMA has the higher performance in terms of BER. On the other hand, both MUSA and PDMA have an identical performance.

In [9], the major technology aspects of non-orthogonal multi-user superposition transmission and shared access were addressed. The discussion covered basic principles and individual schemes for both downlink and uplink cellular systems, for both mobile broadband and Internet-of-things scenarios. Several key techniques were described: SCMA, MUSA and PDMA, together with simulation results. The results as well included the user overloading performance with the BER for the three techniques. In [5], we proposed a new type of spreading codes, 5-ary codes. We compared the performance of 5-ary codes with different complex spreading codes types while changing the ordering method of the SIC receiver for MUSA. We found that Signal to Interference plus Noise Ratio (SINR) based ordering SIC method has better system performance for MUSA while using either 3-ary codes or 5-ary codes.

In this paper, the performance of BER for our proposed 5-ary MUSA scheme and SCMA is studied in terms of user overloading showing the effect of increasing the number of users for the two schemes while keeping the same system parameters. The rest of the paper is organized as follows. In Section 2, 5-ary MUSA with the design of the complex spreading codes, models of MUSA transmitter and receiver and SCMA with the transmitter and receiver models are described. Numerical results are provided in Section 3 and the conclusion is given in Section 4.

\section{RESEARCH METHOD}

In this section we are going to give a brief view on our proposed scheme for multiple access which is 5-ary MUSA showing both transmitter and receiver models including the type of codes used for spreading. Another scheme is also discussed in this section called SCMA showing both the transmitter and receiver models.

\subsection{5-ary Multi User Shared Multiple Access (MUSA)}

MUSA adopts a grant-free access strategy that simplifies the access procedure significantly. In addition, an advanced code domain non-orthogonal complex spreading is used to accommodate massive number of users in the same radio resources. The characteristics of MUSA lie mainly on the system performance improved significantly. It supports a huge number of users-access, and no synchronization is needed which is beneficial to improve the battery life. Therefore, the technology of MUSA uplink is very suitable for the IoT network application where the uplink transmissions are not tightly scheduled, and the grants for transmission are not signaled per user basis, and with a high overloading [10, 11].

Figure 1 shows the basic features of MUSA with $300 \%$ user overloading. Grant-free transmission and high user overloading performance can be achieved by MUSA, which is important to improve connection efficiency and support the required connection density [12]. A family of complex sequences with short length is chosen as spreading sequence for their ability to enable simple and robust successive interference cancellation at the receiver and deal with higher user overload.

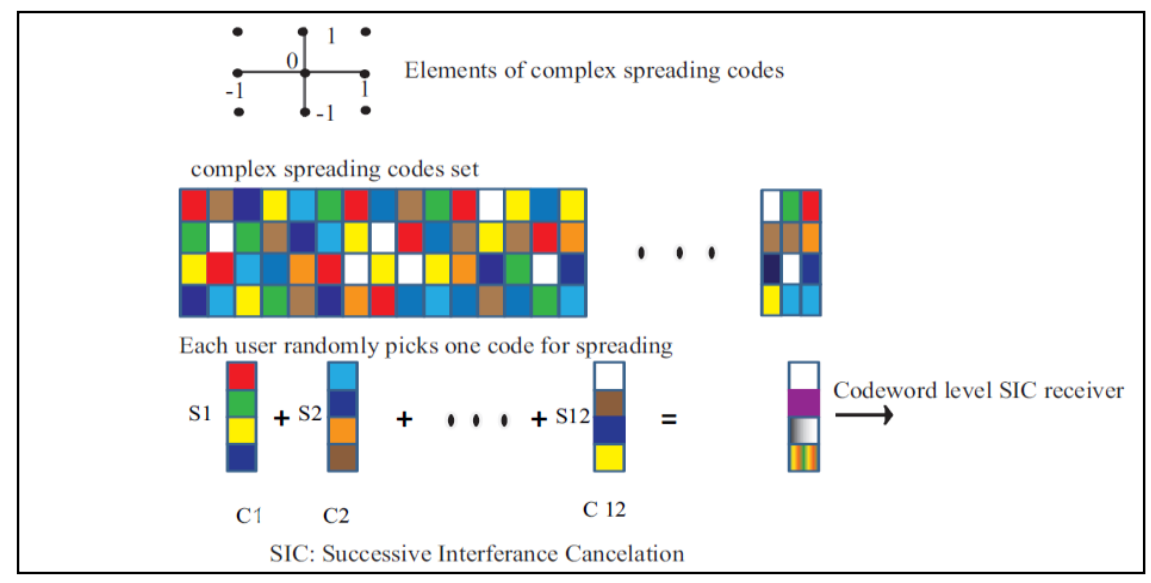

Figure 1. An example of MUSA with user overloading 300\% [11] 
User overloading means having a number of users that is more than the number of available resources and they have to share these resources in order to access the network. For MUSA, each modulated symbol is spreaded by a complex spreading code of length $L$ to be transmitted on $R$ time and/or frequency resources [13]. If the number of accessing users is $\mathrm{N}$, user overloading can be defined as:

$$
\text { useroverloading }=\frac{\mathrm{N}}{\mathrm{L}} \times 100 \%
$$

\subsubsection{5-Ary MUSA Transmitter Model}

In our system model, MUSA transmitter is based on a random process for generating stream of data bits for each user. Each user's data is then modulated using Quadrature Phase Sift Keying (QPSK) giving a stream of symbols for each user; the number of symbols now is half the number of bits. Each user's modulated symbols are then spreaded using a special type of spreading codes known as 5-ary complex spreading code, which will be explained shortly. Blind detection is applied at base station for MUSA in which active user, user spreading code and user channel would not be known before handing [14].

\subsubsection{Spreading Sequences}

Spreading codes with short length and good user overloading performance are designed for MUSA. As the spreading code length is relatively short and its elements have limited values, base station can generate numerous local spreading codes with low correlation. The family of complex spreading codes is very useful, due to the design freedom with real part and imaginary part. Complex Spreading Codes for 5-ary sequence [5] takes their values for each element in the code from the set $\{-2,-1,0,1,2,-1+i, i, 1+i,-1-i,-i, 1-i,-2+i,-2+2 i$, $-1+2 i,-2-i,-2-2 i,-1-2 i, 2 i,-2 i, 1+2 i, 1-2 i, 2+i, 2+2 i, 2-i, 2-2 i\}$. As shown in Figure 2, the set consists of 25 values that will be used to generate the code. As a result, $25^{\mathrm{L}}$ codes could be generated, which may be much better for large number of users accessing in a grant-free manner.

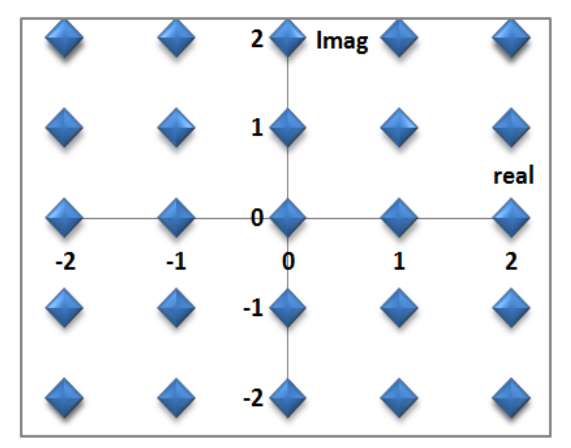

Figure 2. Complex set $\{-2,-1,0,1,2\}(\mathrm{M}-\mathrm{ary}=5)$

\subsubsection{5-ary MUSA Receiver Model}

MUSA receiver is mainly based on SIC, that is used to separate the superimposed symbols according to the received SINR difference. An Ordered Successive Interference Cancellation (OSIC) is one type of the detection methods that is used to reveal all the received data streams as stages. The revealed signal from each stage is deducted from the received signal then the residual signal can be used in the next stages [15] as shown in Figure 3.

In MUSA, SIC is used to fulfill Multi User Detection (MUD) at the receiver. One of the standard linear detection methods is the Minimum Mean Square Error (MMSE) technique and we are going to use it to detect user's data in our model. The model equation for a system with a linear receiver is given by

$$
\mathrm{y}=\mathrm{hx}+\mathrm{n}
$$

where $\mathrm{y}$, is the total received signal at the receiver, $\mathrm{h}$ is the channel coefficient matrix, $\mathrm{x}$ is the composite transmitted signal and $\mathrm{n}$ is a complex-valued noise sample taken from a zero mean Gaussian distribution with variance $\sigma^{2}$.

In order to detect the signal of each user at the receiver, we must first compute the inverse of the channel matrix $\mathrm{h}$ which is $\mathrm{h}^{-1}$ and multiply (2) by this inverse to get the estimated signal $\tilde{\mathrm{x}}$ 


$$
\begin{aligned}
& \mathrm{h}^{-1} \mathrm{y}=\tilde{\mathrm{x}}+\tilde{\mathrm{n}} \\
& \tilde{\mathrm{x}}=\mathrm{h}^{-1} \mathrm{y}-\tilde{\mathrm{n}}
\end{aligned}
$$

where $\tilde{\mathrm{n}}$ is $\mathrm{n}$ multiplied by $h^{-1}$. For the difficulty of computing the term $\mathrm{h}^{-1} \mathrm{y}$ as the inverse only exists for square matrices, a refined definition of an inverse is $\left(h^{\mathrm{H}} h\right)^{-1} \mathrm{~h}^{\mathrm{H}}$ as

$$
\mathrm{W}_{\mathrm{ZF}}=\left(\mathrm{h}^{\mathrm{H}} \mathrm{h}\right)^{-1} \mathrm{~h}^{\mathrm{H}}
$$

where $(.)^{\mathrm{H}}$ denotes the Hermitian transpose operation, $\mathrm{W}_{\mathrm{ZF}}$ is the weight matrix coresponds to zero forcing technique. For MMSE technique, the MMSE weight matrix is given as

$$
\mathrm{W}_{\text {MMSE }}=\left(\mathrm{h}^{\mathrm{H}} \mathrm{h}+\sigma^{2} \mathrm{I}\right)^{-1} \mathrm{~h}^{\mathrm{H}}
$$

where $\sigma^{2}$ is the variance at the receive antenna and I is the identity matrix. Now rewriting (4), we get

$$
\tilde{\mathrm{x}}=\mathrm{W}_{\text {MMSE }} \mathrm{y}
$$

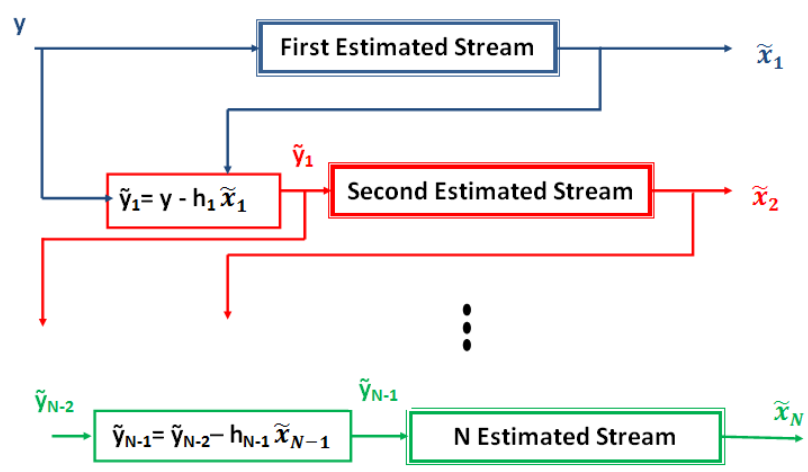

Figure 3. OSIC signal detection

\subsubsection{SINR Based Ordering Method}

In this method, signals having higher Signal to Interference plus Noise Ratio (SINR) are detected first at receiver. The SINR can be calculated using the formula [16]

$$
\operatorname{SINR}_{\mathrm{i}}=\frac{\mathrm{E}_{\mathrm{x}}\left|\mathrm{w}_{\mathrm{i}, \mathrm{MMSE}} \mathrm{h}_{\mathrm{i}}\right|^{2}}{\mathrm{E}_{\mathrm{x}} \sum_{\mathrm{l} \neq \mathrm{i}}\left|\mathrm{w}_{\mathrm{i}, \mathrm{MMSE}} \mathrm{h}_{\mathrm{l}}\right|+\sigma^{2}\left\|\mathrm{w}_{\mathrm{i}, \mathrm{MMSE}}\right\|^{2}}
$$

where $E_{x}$, is the energy of the transmitted signals, $w_{i, M M S E}$ is the ith row of the MMSE weight matrix and $h_{i}$ is the $\mathrm{i}^{\text {th }}$ column of the channel matrix with i referring to the number of transmitting antenna.

\subsection{Sparse Code Multiple Access (MUSA)}

One of the non-orthogonal multiple-access techniques that is being sophisticated with $5 \mathrm{G}$ and other future communications systems is SCMA. In many approaches, SCMA can be treated as a combination of Code Division Multiple Access (CDMA) that is used in $3 \mathrm{G}$ networks and Orthogonal Frequency Division Multiple Access (OFDMA) the one being used in 4G networks [9]. Figure 4 shows an example of SCMA, where the coded data stream are directly mapped to a codeword with nonzero sparse elements from a codebook. Here, there are only 4 orthogonal resources used to transmit 6 sparse codewords giving a user overloading of $150 \%$. Each codeword is a column in the codebook. The way of choosing the codeword in a certain codebook for a certain user depends on the user's coded bits. As an example, if the users coded bits are $(0,0)$, the first column will be the codeword for this data, $(0,1)$ takes the second codeword, $(1,0)$ takes the third codeword and $(1,1)$ takes the last codeword. Take into account that in any codebook the number of codewords and the number of orthogonal resources must be the same. 


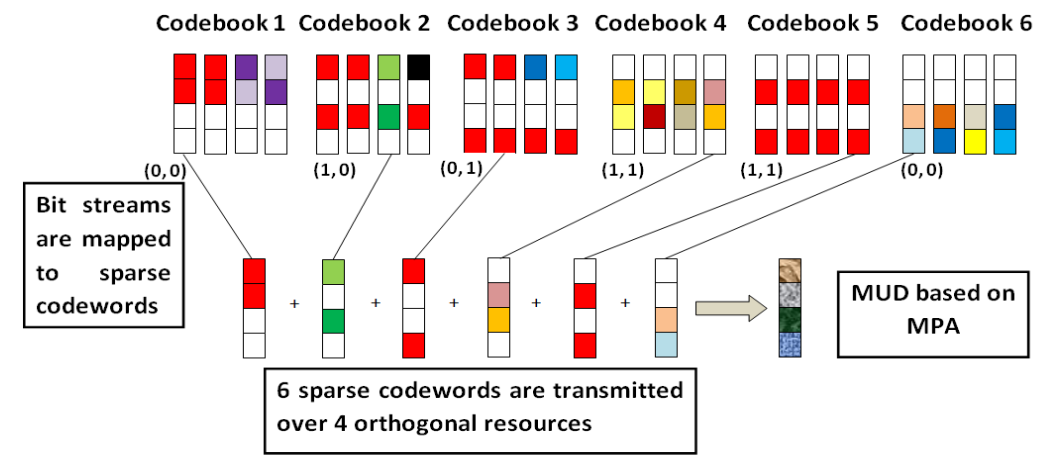

Figure 4. An example of SCMA with six users and $150 \%$ overloading

\subsubsection{SCMA Transmitter Model}

At the transmitter side, each user is allocated a different codebook having a number of codebooks equal to N. Each codebook contains $R$ codewords each one of length $S$. The number of non zero elements (NZE) in each codeword must be less than $S$, leading to a sparse codewords. The received signal on subcarrier $s$ can be represented by:

$$
y_{s}=\sum_{n=1}^{N} h_{s n} x_{s n}+n_{s}
$$

where $\mathrm{x}_{\mathrm{sn}}$ is the $\mathrm{s}_{\mathrm{th}}$ component of the codeword $\mathrm{x}_{\mathrm{n}}$ for user $\mathrm{n}, \mathrm{h}_{\mathrm{sn}}$ is the channel gain of user $\mathrm{n}$ at the $\mathrm{s}_{\mathrm{th}}$ subcarrier, and $n_{k}$ is a complex noise sample taken from a zero mean Gaussian distribution with variance $\sigma^{2}$. The total system model is shown in Figure 5.

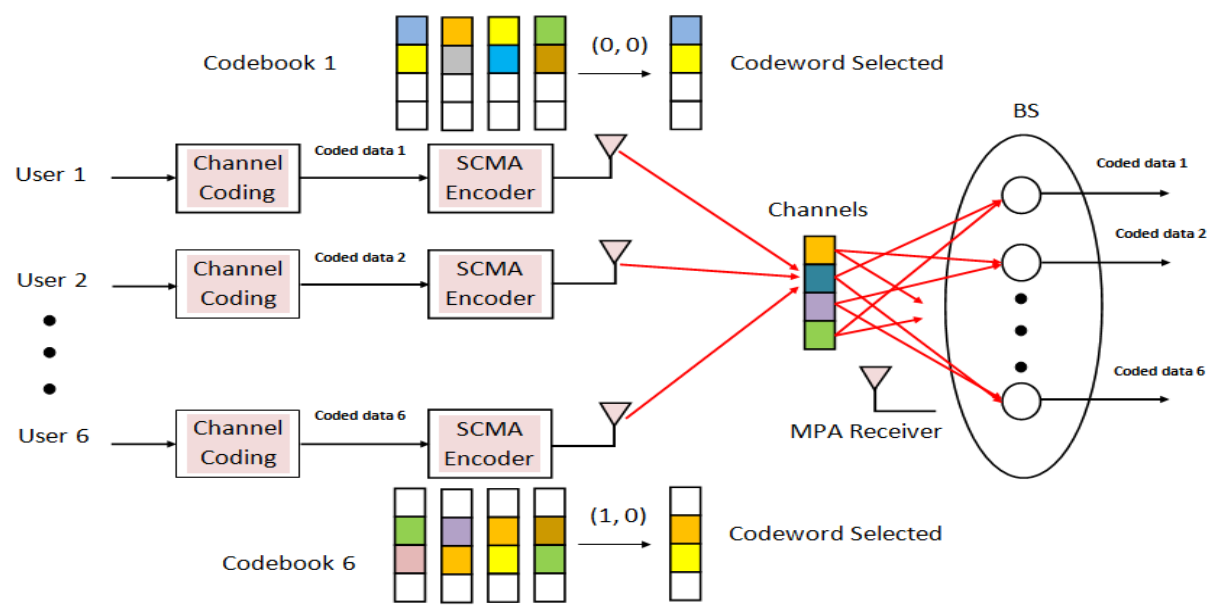

Figure 5. SCMA transmitter and receiver

\subsubsection{SCMA Receiver Model}

The procedure of Message Passing Algorithm (MPA) can be explained by the factor graph as shown in Figure 6; the graph includes both variable nodes and factor nodes. Particularly, in SCMA, variable nodes consist of transmitted codewords from $N$ users, factor nodes can be seen as the received signals over $K$ subcarriers, and there exists an edge between a variable node $x_{n}$ and a factor node $y_{k}$ if and only if $x_{k n} \neq 0$. Message can be passed among variable nodes and factor nodes through the edges. As shown in Figure 6, each variable node is connected only with 2 factor nodes and each factor node can be connected with three variable nodes.

In order to maintain a reasonable level of sparsity, the number of connections between a certain variable node and all factor nodes must not exceed the total number of factor nodes $k$ [17]. 
1) Number of connection between a variable node and all factor nodes $=2$.

2) Number of users $N=\left(\begin{array}{l}K \\ 2\end{array}\right)=\frac{k(k-1)}{2}=6$, for $k=4$

3) User overloading factor $\lambda=\frac{N}{K}=1.5=150 \%$

4) Factor graph $F=\left[\begin{array}{llllll}1 & 1 & 1 & 0 & 0 & 0 \\ 1 & 0 & 0 & 1 & 1 & 0 \\ 0 & 1 & 0 & 1 & 0 & 1 \\ 0 & 0 & 1 & 0 & 1 & 1\end{array}\right]$

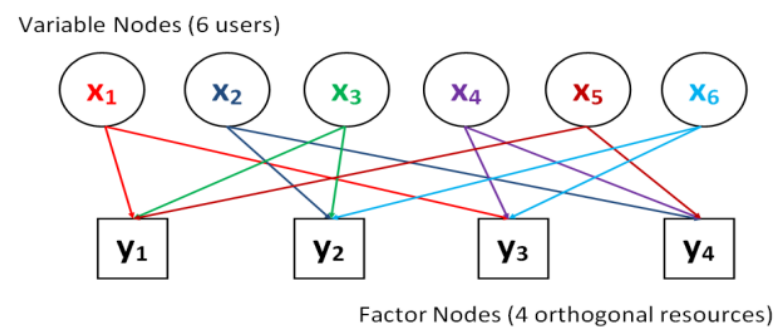

Figure 6. Factor graph representation of MPA

To enable higher connectivity in SCMA, the use of SCMA overloading is very benefit. However, increasing the overloading factor $\lambda$ will cause an increase on the number of colliding layers per each resource element $(R E)$. Superimposing large number of codeword elements at a $R E$ causes a significant degradation of BER performance. The complexity of MPA is proportional to $\lambda$, as increasing $\lambda$ increases the complexity exponentially [18].

\section{RESULTS AND ANALYSIS}

In this section, simulations with small packet traffic model are performed. Particularly, QPSK modulation is used during this simulation. The total number of users is varying between $\{4,6,8,10$, and 12$\}$ sharing 4 orthogonal resources. Therefore, the overloading factor is varying between $\{100 \%, 150 \%, 200 \%$, $250 \%$ and $300 \%$. The SNR takes a range from $0 \mathrm{~dB}$ to $50 \mathrm{~dB}$. The codebooks in SCMA are designed according to [15], spreading sequences in MUSA are generated by pseudorandom sequences whose real and imaginary are taken from $\{-2,-1,0,1,2\}$ for 5 -ary codes and taken from $\{-1,0,1\}$ for 3 -ary codes. The system performance is shown in terms of BER in Rayleigh fading channels.

In Figure 7, the BER performance is shown for 5-ary MUSA, 3-ary MUSA and SCMA is shown for $150 \%$ user overloading. The results show that SCMA has better performance than the other types because of the low user overloading beside the shaping gain property. In addition, 5-ary MUSA performance outperforms the 3-ary MUSA. Comparing this result with the results in [4], we find that the similar results exist of both SCMA and 3-ary MUSA. We added to the graph the performance of 5-ary MUSA which appears better than 3-ary MUSA.

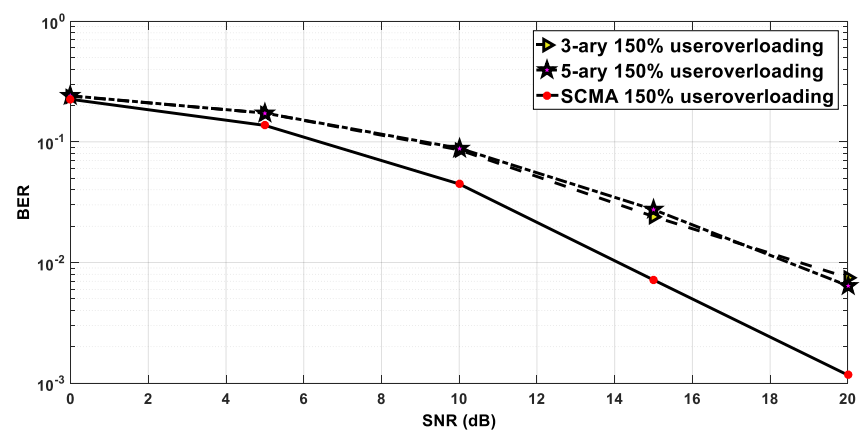

Figure 7. MUSA and SCMA $150 \%$ user overloading 
In Figure 8, the BER performance is shown for $200 \%$ user overloading. The results show that while increasing the number of users that yields to higher overloading, SCMA's performance is affected by the collision that occurs due to the positions of zeros in different codebooks are not distinct. It is shown that 5-ary MUSA has the best performance. It is also expected for the same small number of orthogonal resources and while increasing the number of users sharing them the BER will increase compared to Figure 7.

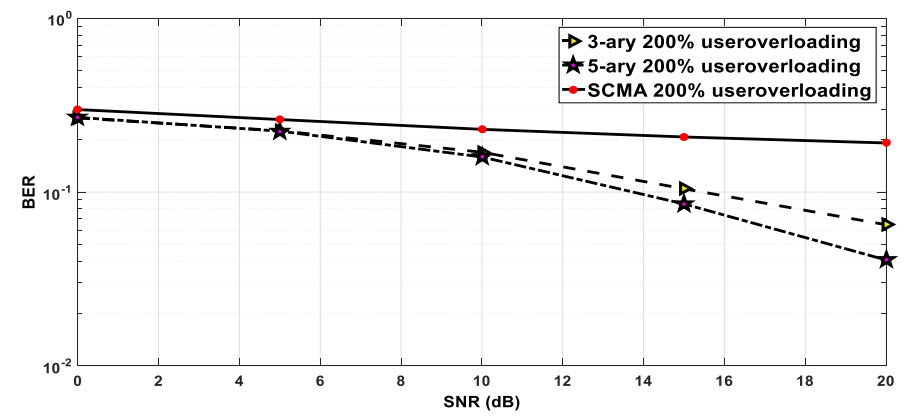

Figure 8. MUSA and SCMA 200\% user overloading

In Figure 9, the BER performance is shown for $250 \%$ user overloading. The results show that SCMA has the lowest performance compared to MUSA even that MUSA's performance decreases but it still outperforms SCMA. In Figure 10, the BER performance is shown for 300\% user overloading. The results show that 5-ary MUSA still has the best performance.

Taking $\mathrm{SNR}=20 \mathrm{~dB}$ as a mid point to study the effect of changing the number of users while using SCMA and MUSA yields to the results shown in Figure 11. As shown, till 150\% overloading, SCMA has better performance than 5-ary MUSA. At 200\% overloading, both types of MUSA has lower BER than SCMA specifically 5-ary one.

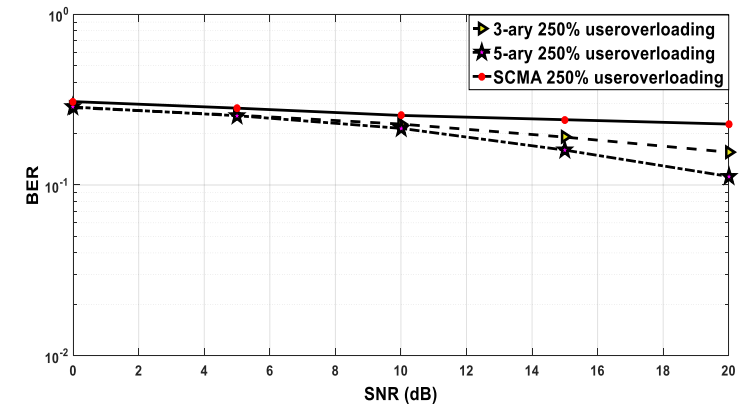

Figure 9. MUSA and SCMA 250\% user overloading

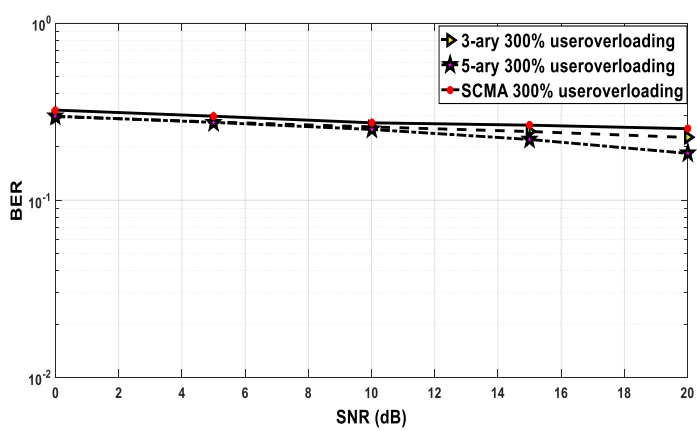

Figure 10. MUSA and SCMA 300\% user overloading

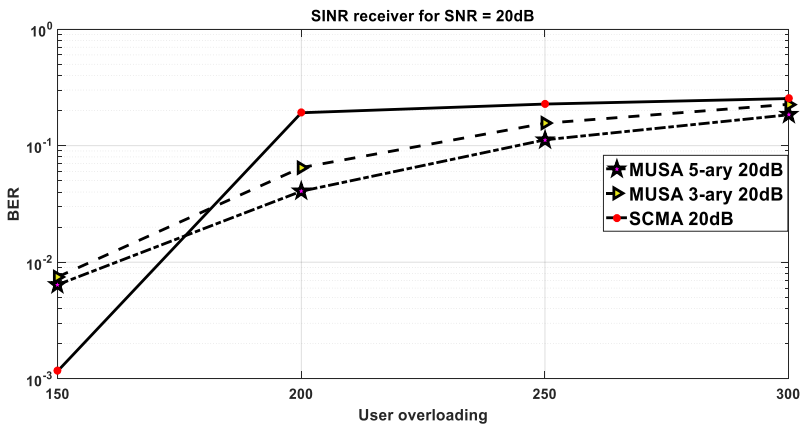

Figure 11. MUSA and SCMA user overloading for $\mathrm{SNR}=20 \mathrm{~dB}$ 


\section{CONCLUSION}

In this paper, we compared the performance of two non-orthogonal multiple access techniques which are SCMA and our proposed scheme 5-ary MUSA while changing the user overloading for different values of SNR. The complex spreading code design is briefly described for the 5-ary MUSA. The process of MPA for SCMA is explained showing the factor graph. The final results show that SCMA is the suitable technique for multiple access for small user overloading as it gives lower BER compared to both types of MUSA. While increasing user overloading, the performance of SCMA decreases and thus 5-ary MUSA gives better performance than SCMA. The BER is increasing for higher user overloading and for the same small number of orthogonal resources, so to improve the results we must use a suitable number of resources that can accommodate the huge number of users in the future for a real communication system.

\section{REFERENCES}

[1] Huawei Whitepaper, 5G: A Technology Vision, Nov. 2013. http://www.huawei.com/ilink/en/download/HW314849.

[2] J. G. Andrews, S. Buzzi, W. Choi, S. V. Hanly, A. Lozano, A. C. K. Soong, and J. C. Zhang, What will 5 G be?, IEEE Journal on Selected Areas in Communications, 32(6), 1065-1082, 2014.

[3] L. Dai, B. Wang, Y. Yuan, S. Han, C. I and Z. Wang, Non-Orthogonal Multiple Access for 5G: Solutions, Challenges, Opportunities, and Future Research Trends, IEEE Communication Magazine, 53(9), 74-81, 2015.

[4] B. Wang, K. Wang, Z. Lu, T. Xie and J. Quan, Comparison Study of Non-Orthogonal Multiple Access Schemes for $5 G$, IEEE Broadband Multimedia Systems and Broadcasting (BMSB), Belgium, 2015.

[5] E. M. Eid , M. M. Fouda, A. S. Tag ELdien and M. M. Tantawy, Performance Analysis of MUSA with Different Spreading Codes Using Ordered SIC Methods, IEEE International Conference on Computer Engineering and Systems (ICCES), Egypt, 2017.

[6] T. Yunzheng, L. Long, L. Shang, et al., A Survey: Several Technologies of Non-Orthogonal Transmission for 5G, in China Communications, 12(10), 1-15, 2015.

[7] H. Nikopour and H. Baligh, Sparse Code Multiple Access, IEEE International Symposium on Personal, Indoor and Mobile Radio Communications (PIMRC), UK, 2013.

[8] M. Taherzadeh, H. Nikopour, Alireza Bayesteh, and Hadi Baligh, SCMA Codebook Design, IEEE Vehicular Technology Conference (VTC Fall), Canada, 2014

[9] F.-L. Luo and C. J. Zhang, Signal Processing for 5G: Algorithms and Implementations. NY, USA: Wiley, 115-142, 2016.

[10] F. Hu, D. Xie and S. Shen, On the Application of the Internet of Things in the Field of Medical and Health Care, IEEE International Conference on Green Computing and Communications and IEEE Internet of Things and IEEE Cyber, Physical and Social Computing, Beijing, China, 2013.

[11] N. Kumar, N. Chilamkurti and S. C. Misra, Bayesian Coalition Game for the Internet of Things: an Ambient Intelligence-based Evaluation, IEEE Communications Magazine, 53(1), 48-55, 2015.

[12] 3GPP, R1-166403, Grant-free multiple access schemes for mMTC, 2016.

[13] Z. Yuan, G. Yu, W. Li, Y. Yuan, and X. Wang, Multi-User Shared Access for Internet of Things, IEEE Vehicular Technology Conference, China, 2016.

[14] 3GPP, R1-166404, Receiver details and link performance for MUSA, 2016.

[15] G. J. Foschini, Layered Space-Time Architecture for Wireless Communication in a Fading Environment When Using Multi-Element Antennas, Bell Labs Technical Journal, 1(2), 41-59, 1996.

[16] Y.Cho, J.Kim, W.Yang, and C. Kang, MIMO-OFDM Wireless Communication with Matlab, John Wiley \& Sons (Asia) Pte Ltd, 322-324, 2010.

[17] Z. Bai, B. Li, M.Yang, Z. Yan, X. Zuo and Y. Zhang, FH-SCMA: Frequency-Hopping Based Sparse Code Multiple Access for Next Generation Internet of Things, IEEE Wireless Communications and Networking Conference (WCNC), USA, 2017.

[18] M. Alam and Q. Zhang, Performance Study of SCMA Codebook Design, IEEE Wireless Communications and Networking Conference (WCNC), USA, 2017. 